\title{
GAUSSIAN-PROFILE BEAMS
}

\section{Edward $P$, Lee}

UCID--19639

Collaboratorș: G. J. Caporaso, F. W. Chanibers,

A. G. Cole, W. M. Fawley and A. C. Hindmarsh

November 3; 1982

\section{Lawrence Livermore National Laboratory \\ University of California \\ Livermore, CA 94550}

\section{ABSTRACT}

The growth rate of the hose instability is derived for a beam with Gaussian radial profile, using the spread mass model of phase mix damping. It is found that the maximum growth rate of a convecting wave packet is $49 \%$ iarger than that derived for a beam with the Bennett profile, and the inverse group velocity $(d z / d \tau)$ is also increased by about this amount.

A general discussion of spread mass models is presented along with an explangition of the regurgitation phenomena seen in their numerical treatment.

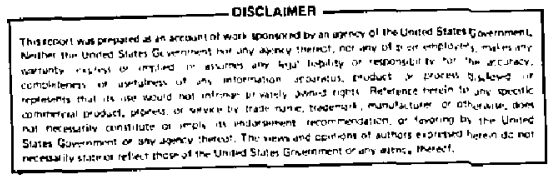

Work performed jointly under the auspices of the U. S. Department of Energy by Lawrence Livermore National Laboratory under contract W-7405-ENG-48 and for the Department of Defense under Defense Advanced Research Projects Agency ARPA Order No. 4395 A\#1, monitored by Naval Surface Weapons Center under document number N60921-82-WR-W0056. 


\section{Introduction}

The Bennett profile has most often been adopted for the analys is of pinched beam dynamics because theory ${ }^{1}$ and experiment ${ }^{2}$ have established that it is generated by multiple scattering in uniform density gas. However, the Gaussian profile

$$
J_{b}(r)=\frac{I_{b}}{\pi a^{2}} \exp \left(-r^{2} / a^{2}\right),
$$

has also been used, especially for numerical computations, for several reasons:

(a) The scattered (Bennett) profile develops only over a scale distance which is long compared with a betatron wavelength; the Guassian may be a better representation of the initial profile.

(b) It is often convenient for computations to use a profile with a more raid fall-off at large $r$ than the Bennett.

(c) The sensitivity of instability growth rates to the assumed profile should b 2 examined.

The present report is a compilation of results derives for the Gaussian profile, whigh are conisared with those derived for the Bennett. There is also a summary of the spread mass model features for general profi.le, whase detailed justification is given in a paper ${ }^{3}$ by the author and is not repeat. here.

It has been known for some time that phase mix damping by mass spread tends to undo itself at large values of $k_{B} z$. This is called the "regurgitation phenomena" and has not previously been explained. During the present study some good graphical examples of regurgitation were obtained which showed interesting new features (such as beating). This inspired a partial explanation of regurgitation, which is presented in Section VII. 
II. Normalization

Since the growth rates due to differing current profiles are to be compared, some conventions inust be adopted to make sure that factors other than profile are not varied, Let the net beam current $\left(I_{b}\right)$ and beam current density on-axis $\left[J_{b}(0)\right]$ be the same for all profiles considered. Then a scale radius (a), defined by

$$
I_{b}=\pi a^{2} J_{b}(0),
$$

is also the same for all profiles. The rms radius $(R)$ does depend on profile, but that is no great loss since it has proved to be an unreliable diagnostic in simulation, 4 and it is infinite for the Bennett profile.

Particle charge (q) and moment $\mathrm{m}$ (Brmc) are fixed, so the maximum betatron frequency for all profiles is given by the on-axis value

$$
k_{m}^{2}=\frac{2 \pi q J_{b}(0)}{B \gamma m C^{3}}=\frac{2 I_{b}}{a^{2} I_{A}} \text {, }
$$

where

$$
I_{A}=B \operatorname{MBC}^{3}\left\{a \approx\left\{17 k A_{1}\right\}\left(\frac{m e}{m_{e}^{q}}\right) B \gamma\right.
$$

is the Alfven current.

The effective mean betatron frequency for hose motion (sometimes called the "shaking frequency") is the profile-dependent quantity

$$
\begin{aligned}
k_{s}^{2} & =\left(\frac{q \pi}{\beta r m c^{2} I_{b}}\right) \int d r r \frac{\partial J_{b}}{\partial r} \frac{\partial A_{0}}{\partial r} \\
& =k_{m}^{2} \int \frac{d r 2 r}{a^{2}}\left(\frac{\pi a^{2} J_{b}}{I_{b}}\right)^{2} .
\end{aligned}
$$


For the Gaussian profile $k_{s}^{2}=k_{m}^{2} / 2$. We do not consider the effect of return current in the definitions of $k_{s}$ and $k_{m}$, although it is implicit in the 2 - d formulation given in Section III and mast numerical treatments. Here the quantity $\left(k_{m} / k_{s}\right)^{2}$ is derived for a given profile without return current, and is considered to be a measure of profile roundedness. A sumary of profile dependent quantities is given in Table 1.

Electrical conductivity $\sigma(r)$ is given a profile which may differ from

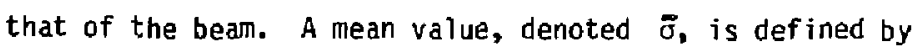

$$
\bar{\sigma} \pi a^{2}=\int_{0}^{\infty} d r 2 \pi r \sigma(r),
$$

which is held constant in the present study. We usually use the same profile for $\sigma(r)$ and $J_{b}(r)$ in analytical work; this has some justification at high gas pressures, where avalanching and conductivity spreading mechanisms are minimal. Code work to date indicates this assumption is valid for $I_{b} \leq 10 \mathrm{kA}$ and $P\left(N_{2}\right) \geq 200$ torr. We therefore have $\sigma(r=0)=\bar{\sigma}$, e.g., for Gaussian profiles,

$$
\sigma(r)=-\bar{\sigma} \exp \left(-r^{2} / a^{2}\right)
$$

The characteristic dipole decay time $\left(\tau_{7}\right)$ is well approximated by the variational expression ${ }^{3}$

$$
\begin{aligned}
\tau_{1} & =\left[\int \frac{d r}{r} \sigma(r)\left(\frac{I_{r}}{c}\right)^{2}\right]\left[d r r \pi J_{b}^{2}\right]^{-1} \\
& =\frac{k_{m}^{2}}{k_{s}^{2}} \int \frac{d r}{r} \frac{2 \pi \sigma(r) a^{2}}{c^{2}}\left(\frac{I_{r}}{I_{b}}\right)^{2},
\end{aligned}
$$




$$
-5-
$$

where

$$
I_{r}=\int_{0}^{r} d r^{\prime} 2 \pi r^{\prime} J_{b}\left(r^{\prime}\right) .
$$

For Gaussian $J_{b}(r)$ :

$$
I_{r}=I_{b}\left[1-\exp \left(-r^{2} / a^{2}\right)\right] \text {, }
$$

and assuming Gaussian $\sigma(r)$,

$$
\tau_{1}=\left[2 \ln \left(\frac{4}{3}\right)\right]\left(\frac{\pi \bar{\sigma} a^{2}}{c^{2}}\right)=(.575)\left(-\frac{e^{2}}{c^{2}}\right) .
$$

We oenote this value by $\tau^{G}$, and the similarly derived values for the flat and Bennett profiles are denoted $\tau^{F}$ and $\tau^{B}$. 


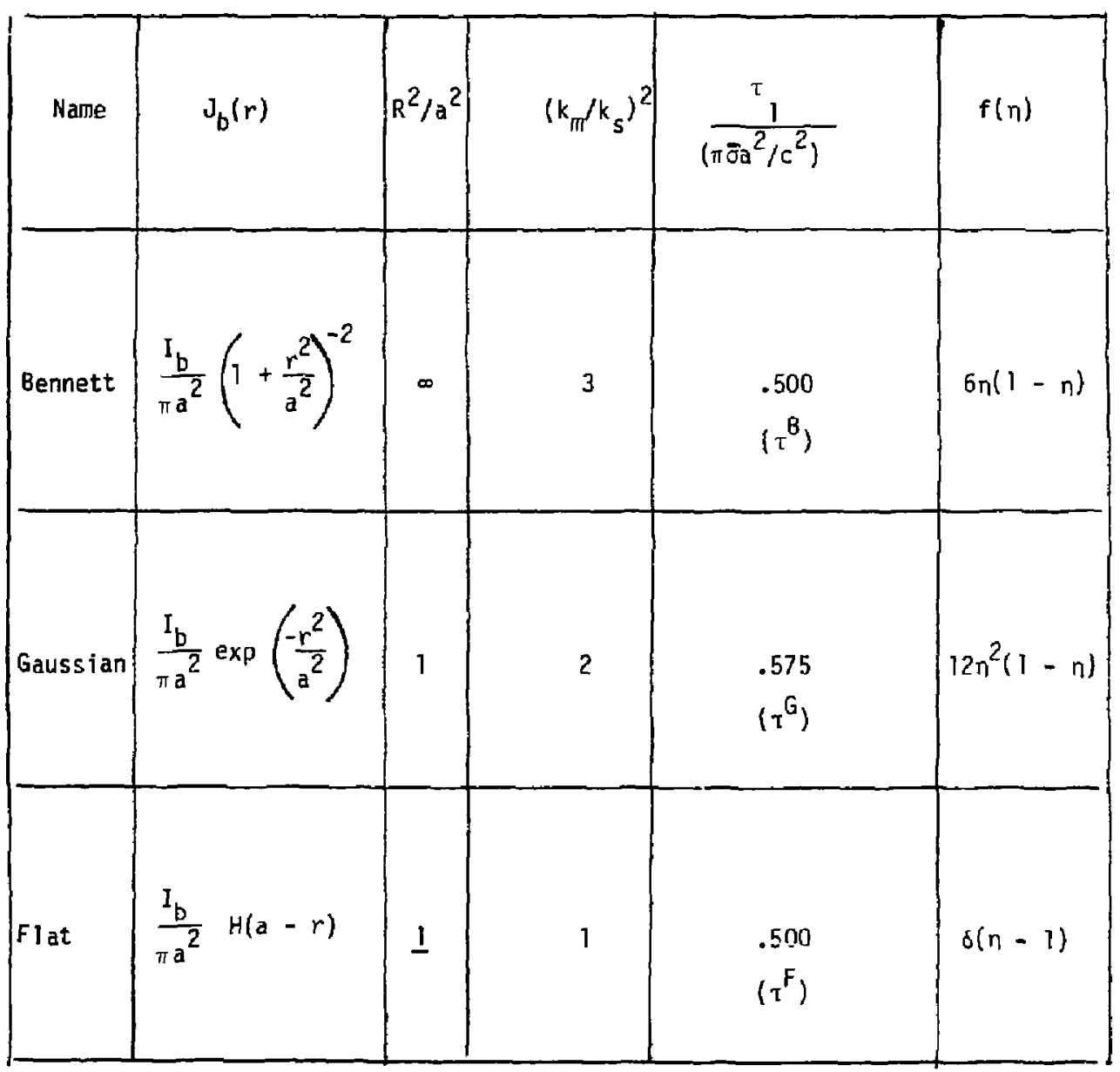

Table 1: Summary of Profile Dependent Quantities 


\section{Spread Mass Model}

The rigid-beam hose equation is modified by spreading the shaking frequency $\left(k_{s}\right)$ over the range $\left(0 \leq k \leq k_{m}\right)$. Let $Y_{n}$ be the transverse displacement associated with frequency

$$
k_{n}=\sqrt{n} k_{m}
$$

where $0 \leq n \leq 1$. Then the equation of motion for $Y_{\eta}$ is taken to be

$$
\begin{gathered}
\frac{d^{2} Y_{n}}{d z^{2}}=n\left(\frac{k^{2}}{k^{2}}\right)\left(\frac{q \pi}{s r m c^{2}}\right) \int \frac{d r r}{I_{b}}\left[-Y_{\eta} \frac{\partial J_{0}}{\partial r} \frac{\partial A_{0}}{\partial r}\right. \\
\left.+\frac{J_{b_{0}}}{r} \frac{\partial}{\partial r} r A_{\eta}\right],
\end{gathered}
$$

with $A_{0}$ and $A_{1}$ satisfying the field equations (in the "magnetic regime")

$$
\begin{aligned}
& \frac{1}{r} \frac{\partial}{\partial r} r \frac{\partial A_{0}}{\partial r}-\frac{4 \pi \sigma_{0}}{c^{2}} \frac{\partial A_{0}}{\partial \tau}=-\frac{4 \pi}{c} J_{b_{0}}, \\
& \frac{\partial}{\partial r} \frac{1}{r} \frac{\partial}{\partial r} r A_{1}-\frac{4 \pi}{c^{2}}\left(\sigma_{0} \frac{\partial A_{1}}{\partial \tau}+\sigma_{1} \frac{\partial A_{0}}{\partial \tau}\right)=-\frac{4 \pi}{c} J_{b_{1}} .
\end{aligned}
$$

The perturbed component of current density is given by

$$
J_{b_{1}}=-\langle\gamma\rangle \frac{{ }^{\partial J_{b_{0}}}}{\partial r} \text {, }
$$

where $\varepsilon Y>$ is an appropriately weighted average of $Y_{n}$ :

$$
\langle Y\rangle=\int_{0}^{l} d n f(n) \gamma_{n}
$$


The problem is to specify a weight function $f(n)$ which yields phase mix damping appropriate for a particular profile $J_{b}(r)$. Several criteria for $f$ must be satisfied:

(a)

$$
\int_{0}^{1} d n f(n)=1 \text {, }
$$

$$
\begin{aligned}
& f(n) \geq 0, \\
& f(n \rightarrow 0)=0, \\
& \int_{0}^{1} \operatorname{dn} \frac{f(n)}{n}=\frac{k_{m}^{2}}{k_{s}^{2}},
\end{aligned}
$$

(e)

$$
f(n) \text { is simple. }
$$

If condition (c) is not met, then the hose mode may be stabilized (unphysically) by the excess of low frequency oscillators. Condition (d) is required in order to reproduce the low frequency $1 \mathrm{in}^{-1} \mathrm{t}$ of hose growth, which is an exact result from kinetic theory.

It may also be required that

$$
f(n+l)=0,
$$

in order to reproduce phase mix damping rates determined from numerical simulation. This point has not yet been clearly established -- due to the very large required number of simulation particles. Further, the detailed dependence of $f$ on $n$ as $\eta+1$ may depend on profile and aiso be inferred from simulation. More work is required here.

A non-unique function which satisfies (a) - (f) for the Gaussian profile

$$
\left.[\text { recal }] k_{m}^{2} / k_{s}^{2}=2\right] \text { is }
$$




$$
f^{G}(n)=12 \pi^{2}(1-n) \text {. }
$$

The corresponding functions for the Bennett and $f$ lat profiles are given in Table 1.

\section{Dispersion Relation}

A further set of approximations reduces the coupled equations for fields and beam displacements $\left(Y_{\eta}\right)$ to a single $l$ - $d$ equation for displacements. Essentially, the undisturbed state of the beam is taken to be independent of $z$ and $\tau$, and only the fundamental radial mode of $A_{1}$ is excited. The $Y_{\eta}$ satisfy

$$
\frac{\partial^{2} Y \eta}{\partial z^{2}}=n k_{m}^{2} \int_{-\infty}^{\tau} \frac{d \tau^{\prime}}{\tau_{1}} \exp \left(\frac{\tau^{-}-\tau}{\tau}\right)\left[\left\langle Y^{\prime}\right\rangle-Y_{\eta}\right] .
$$

For the single Fourier mode

$$
Y_{n}=\exp (-i \Omega z-i \omega \tau),
$$

Eq. (25) yields the dispersion relation

$$
-i \omega \tau_{T}=\left\langle\frac{\Omega^{2}}{n k_{m}^{2}-\Omega^{2}}\right\rangle=\int_{0}^{1} \frac{\operatorname{dnf}(n) \Omega^{2}}{n k_{m}^{2}-s^{2}} .
$$

The integral appearing in Eq. (27) is evaluated using the Gaussian weight [Eq. (24)] to obtain the explicit form 
where

$$
-i \omega \tau_{1}^{G}=12 s\left\{\begin{array}{l}
(1-s) s^{2}\left[\pi i+\ln \left\{\left(\frac{1-5}{s}\right)\right]\right. \\
\left.+\left(\frac{1}{6}+\frac{s}{2}-s^{2}\right)\right\}
\end{array}\right.
$$

$$
s=s^{2} / k_{m}^{2} \text {. }
$$

For comparison, the oiennett and flat profjle dispersion relations are

$$
\begin{aligned}
-i \omega \tau_{1}^{B} & =6 s\left\{(1-s) s\left[\pi i+\ln \left(\frac{1-s}{s}\right)\right]\right. \\
& \left.+\left(\frac{1}{2}-s\right)\right\}, \\
-i \omega \tau_{l}^{F} & =\frac{s}{1-s} .
\end{aligned}
$$

The function $\ln [(]-s) / s]$ is taken to be real for $\Omega$ real and in the interval $\left(0<s<k_{m}\right)$, and the branch cuts run downwards in the $s i$ plane from from the points $s t=\left(0, \pm k_{m}\right)$. For other points on the real $s$ axis we have

$$
\left[\pi i+\ln \left(\frac{l-s}{s}\right)\right]=\left\{\begin{array}{cl}
-\pi i+\ln \left|\frac{1-s}{s}\right| & \left(-k_{m}<s i<0\right) ; \\
\ln \left|\frac{s-1}{s}\right| & ,|\Omega|>k_{m} .
\end{array}\right.
$$

\section{Growth Rates and Group Velocity}

The growth rate of the hose mode is inferred from $\mathrm{Eq}$. (28) for the Gaussian profile, or from some other dispersion relation [such as Eq. (30)] for a different model or profile. The easiest thing to do is to tabulate values of $\omega$ for given real $\Omega$; see Figure 1. The maximum imaginary component of $\omega$ obtained in this way gives the growth rate in $t$ of a 
wave packet moving backwards in the beam frame. This is a good picture of mode growth in a short pulse propagating a long distance in $z$. Designating by subscript (o) chis point of maximum imaginary $\omega$, we find

$$
\begin{aligned}
I_{m}\left(\omega_{0}\right) & =1.18 / \tau_{1}^{G}, \\
\operatorname{Re}\left(\omega_{0}\right) & = \pm 1.50 /_{1}{ }^{G}, \\
s_{0} & =\mp .637_{m}, \\
s_{0} & =\left(s i_{0} / k_{m}\right)^{2}=.406 .
\end{aligned}
$$

The (inverse) group velocity at the maximum is given by

$$
\begin{aligned}
\frac{d z}{d \tau} & =-\left(\frac{\partial R_{e} \omega}{\partial \sqrt{2}}\right)_{0}=\frac{12 \pi}{k_{m} \tau^{G}}\left(6 S_{0}^{\frac{5}{2}}-8 S_{0}^{\frac{7}{2}}\right) \\
& =\frac{10.9}{k_{m} \tau^{G}} .
\end{aligned}
$$

For the flat profile, the maximum growth rate is infinite due to the resonance at $s l= \pm k_{m}$, and the wave packet analysis cannot be carried out in the above given form. A comparison between the similar results for the Gaussian and Bennett profiles is

$$
\begin{aligned}
& \frac{\left(I_{m}{ }^{1}{ }_{0}\right)^{G}}{\left(I_{m} \omega_{0}\right)^{B}}=\frac{1.18 \cdot 1^{G}}{.690 / \tau_{1}{ }^{B}}=\left(\frac{1.18}{.690}\right)\left(\frac{.500}{.575}\right)=1.49, \\
& \frac{(\mathrm{d} 2 / \mathrm{d} \tau)^{G}}{(\mathrm{~d} 2 / \mathrm{d} \tau)^{B}}=\frac{\left(10.9 / \mathrm{k}_{\left.\mathrm{m}^{\tau}{ }^{G}\right)}{ }^{\mathrm{G}}\right)}{\left(6.34 / \mathrm{k}_{\left.\mathrm{m}^{\tau}{ }^{B}\right)}{ }^{B}\right.}=\left(\frac{10.9}{6.34}\right)\left(\frac{.500}{.575}\right)=1.49 .
\end{aligned}
$$

Crudely we expect that if the Bennett profile pulse is $49 \%$ Tonger than the equivalent Gaussian, the same saturated hose amplitude will appear at the tail. 
A long pulse (large $\left.\tau / \tau_{1}\right)$, which propagates only a short distance (2), is realistically treated by considering a perturbation with given real w. In that case the dispersion relation must be solved numerically for $s$, whose maximum imaginary component gives the expected growth in $z$ (if a broad range of $\omega$ is present at $z=0$ ). Two solutions are abtained for each real value of $w$, only one of which is unstable, and instability lies only within a finite range of $\omega$-- see Table 2. The point of maximum growth for the Gaussian is

$$
\begin{aligned}
I_{\mathrm{m}}(s) & =(.233) \mathrm{k}_{\mathrm{m}}, \\
\operatorname{Re}(\Omega) & =\mp(.430) \mathrm{k}_{\mathrm{m}}, \\
\omega & = \pm .500 / \tau_{1}^{\mathrm{G}} .
\end{aligned}
$$

The mode is stable for

$$
|\omega|>3.50 / \pi_{1}^{G}
$$

The existence of this "cutaff" is corinected with the presence of phase mix damping and does not occur for the flat profile.

The discussion given in this section is very brief, however, a full account of the wave packet and related analysis is contained in ref. 3. 


\begin{tabular}{|c|c|c|c|c|}
\hline \multirow[b]{2}{*}{$\omega \tau_{1}^{G}$} & \multicolumn{2}{|c|}{ Stable Root } & \multicolumn{2}{|c|}{ Unstable Root } \\
\hline & $\operatorname{Re}\left(s i / k_{m}\right)$ & $I_{m}\left(s i / k_{m}\right)$ & $\operatorname{Re}\left(\Omega / k_{m}\right)$ & $I_{m}\left(\Omega / k_{m}\right)$ \\
\hline 0.0 & 0.000 & 0.000 & 0.000 & 0.000 \\
\hline 0.1 & .769 &. .138 & -.169 & .147 \\
\hline 0.2 & +232 & -.166 & -.252 & .193 \\
\hline 0.3 & .271 & -.181 & -.320 & .218 \\
\hline 0.4 & .298 & -.192 & -.378 & .230 \\
\hline 0.5 & .319 & -.202 & -.430 & .233 \\
\hline 0.6 & .336 & -.211 & -.475 & .230 \\
\hline 0.7 & .351 & -.218 & -.513 & .224 \\
\hline 0.8 & .363 & -.225 & -.547 & .215 \\
\hline 0.9 & .374 & -.231 & -.576 & .204 \\
\hline 1.0 & .384 & -.237 & -.601 & .193 \\
\hline 1.5 & .421 & -.261 & -.686 & .135 \\
\hline 2.0 & .447 & -.279 & -.732 & .0883 \\
\hline 2.5 & .457 & -.295 & -.761 & .0519 \\
\hline 3.0 & .483 & -.308 & -.781 & .0236 \\
\hline 3.5 & .496 & -.319 & -.796 & .000 \\
\hline
\end{tabular}

Tabie 2 Growth Rate for Given Real w. 
VI. Phase Mix Damping

A single perturbed segment of a rounded profile beam acts like a damped oscillator. The spread mass model was motivated by the desire to incorporate this behavior into phenomenological codes, with corroboration from particle simulation. It is therefore of interest to compute the behavior of a single perturbed segment using the spread mass model. Starting from unit amplitude we have

$$
\begin{aligned}
& \langle\gamma\rangle=\int_{0}^{1} \operatorname{dnf}(n) \cos \left(\sqrt{\pi} \mathrm{k}_{m^{2}}\right) \\
& =\frac{24}{2^{6}}\left[\left(-22^{4}+1502^{2}-2400+\frac{5040}{2^{2}}\right) \cos (?)\right. \\
& +\left(222^{3}-7202+\frac{5040}{2}\right) \sin (2) \\
& \left.-120\left(1+\frac{42}{2^{2}}\right)\right]
\end{aligned}
$$

where we define $Z=k_{m} z$. Figure 2 is a graph of this solution taken out to $z=25$. Asymptotic values are

$$
\begin{aligned}
& \langle Y(Z+0)\rangle=1-\frac{3 Z^{2}}{10}, \\
& \langle Y(Z+\infty)\rangle=-\frac{48 \cos (Z)}{Z^{2}} .
\end{aligned}
$$

The latter limit is easily obtained by setting $\eta=x^{2}$ and integrating by parts twice in Eq. (39) [A. C. Hindmarsh]. The first minimum is $\langle\gamma\rangle=-.85$, which can be compared with the more strongly damped Bennett, which has $\langle\gamma\rangle=-.76$. 
Unfortunately, at present chere is no comparison with simulation over long enough $z$ to allow a verification of the $Z^{-2}$ asymptotic dependence. If such a behavior were estab1ished it would reduce the ambiguity in the selection of $f(n)$. Consider the general form [first examined by G. Caporaso].

$$
f(n)=\frac{(n+m+1) !}{n ! m !} n^{n}(1-n)^{m},
$$

where $n$ and $m$ are taken to be positive integers in order to get a simple dispersion relation. To satisfy condition (d) on $f_{\eta}$ [see Eq. (21)], $n$ and $m$ must be related by

$$
m+1=n\left(\frac{k_{n}^{2}}{k_{s}^{2}}-1\right)=0 .
$$

There are an infinity of solutions to Eq. (44). This ambiguity would be eiminated if the decay rate at large $Z$ could be imposed as an additional condition. For the general distribution [Eq. (43)] it can be shown that for

$$
\begin{aligned}
& <y>\propto \frac{1}{z^{m+1}} \quad \text { if } 2(n+1)>m+1 . \\
& \langle y\rangle \propto \frac{1}{Z^{2(n+1)}} \text { if } 2(n+1)<m+1 .
\end{aligned}
$$

To show the seriousness of the ambiguity in $f(\eta)$, note that

$$
f=2 n \quad(0 \leq n \leq 1)
$$

actually satisfies conditions (a) - (e) for the Gaussian. The resultant dispersion relation is simpler than Eq. (28) and the growth rate is somewhat lower [see Appendix]. However, the asymptotic damping rate for a single segment is (only) 


$$
\langle Y(Z+\infty)\rangle=\frac{4 \sin Z}{Z} \text {, }
$$

which may be slow enough to be excluded by comparison with simulation.

\section{The Discrete Case - Regurgitation}

Currently, the EMPULSE code ${ }^{5}$ discretizes $n$ in $N$ even steps

(although A. Hindmarsh suggests there may be a more efficient distribution):

$$
\eta_{i}=\left(i-\frac{7}{2}\right) / N(i=1,2, \ldots, N) \text {, }
$$

and the weights are

$$
f_{i}=\frac{12}{N\left(1+\frac{T}{2 N^{2}}\right)} n_{i}^{2}\left(1-\eta_{i}\right) .
$$

We have (exactly) the desired reiations

$$
\begin{aligned}
& \sum f_{i}=1, \\
& \sum \frac{f_{i}}{n_{i}}=2,
\end{aligned}
$$

and the mean displacement is

$$
\langle\gamma\rangle=\sum_{1}^{N} f_{i} y_{i}(z, \tau) .
$$

Typically, $N=100$ has been used. This would seem at first sight to be a very low value since noise at the $1 / r \bar{N}=10 \%$ level is expected. What is actually observed is damping to the $\approx 1 \%$ level, followed by "regurgitation" to a high level. The code cannot be trusted when run into the regurgitation zone, but this may be quite far out in $z$. 
Figure 3 is an $N=30$ representation of the simple phase mix damping from unit initial amplitude:

$$
\langle Y\rangle=\sum_{1}^{N} f_{i} \cos \left(\sqrt{n_{i}} 2\right) .
$$

Note the features:

(a) The envelope of oscillations regurgitates with a beat-like structure, gradually turning into noise.

(b) The low damped minimum.

(c) The increasing frequency as the envelope approaches a maximum.

(d) Initial damping is in accord with the continuous limit Figure 2.

(e) Regurgitation reaches $\approx 40 \%$ initial amplitude at $z \approx 320$.

(f) Collapse of regurgitation is very sudden -- there is a second minimum at $Z \approx 400$.

The case of $N=100$, run out to $Z=1000$, is disp]ayed in Figure 4 . Again the initial decay goes to a very low level and is in accord with the continuous limit. Here the envelope maximum is reached just beyond $z=1000$ and is only $\approx 20 \%$ of the initial ampitude.

An explanation of some of the regurgitation phenomena, developed by F. Chambers and myself, follows. We wish to evaluate the sum of $N$ oscillators [Eq. (52)] for large 2 . The phase of the oscillator (or disk) with Iable (i) is about

$$
\theta_{i}=\sqrt{\frac{i}{N}}^{-} z
$$

For small $Z$ the $\theta_{i}$ are closely spaced (assuming $N$ is large) and we expect the continuous limit to apply. However, as $Z$ becomes large, a 
subgroup of disks will have phases differing by $2 \pi$ (as $i$ increases by one unit). Later there will be subgroups with phase differences of $4 \pi$, $6 \pi$, etc. as $i$ increases by one unit. Consider the case of phase increment $2 \pi m$ where $m$ is a positive integer. The value of $i$ for which there is an increment of $2 \pi \mathrm{m}$ (denoted $\bar{i}$ ) is approximately the solution of

$$
2 \pi m=\left(\frac{\partial}{\partial i} \sqrt{\frac{i}{N}} \quad z\right)_{i}=\frac{2}{2 \sqrt{i N}} .
$$

Hence the "m th phase group" consists of values of $i$ near

$$
\bar{i}(m, 2)=\left(\frac{2}{4 \pi m \sqrt{N}}\right)^{2}
$$

at the distance $Z$.

The important question is "how many disks are in phase"? If $\bar{i}$ is much larger than $N$ or is very small compared with $N / 2$, then there are essentially no disks in the $m$ th phase group. To answer this question more accurately, let $\delta i$ denote the increment of $i$ away from $j$ corresponding to a phase increment of $\pi / 4$; the range of $i$ values which are in the phase group is roughly $2 \delta i$. To obtain $\delta i$ we therefore solve the relation

$$
\frac{\pi}{4}=-\delta i\left(\frac{1}{2} \frac{\partial^{2}}{\partial i^{2}} \sqrt{\frac{i}{N}} z\right)_{i}=\frac{\delta i z}{8 \sqrt{N i}} .
$$

We have:

$$
\left(\begin{array}{c}
\text { No. of disks } \\
\text { in phase }
\end{array}\right) \approx(2 \delta j)=\frac{4 \pi}{2} \sqrt{N_{i}^{3}}=\frac{i}{m},
$$

where we have used Eq. (55) to eliminate $z$. 
-19-

The value of the sum [Eq. (52)] should be approximately (for the $m$ th phase group)

$$
\langle Y\rangle_{m} \approx f_{i} \cos \left(\sqrt{\frac{\bar{j}}{N}} 2\right) \quad(2 \delta i)\left(\frac{N}{N+i}\right),
$$

where the factor $N /(N+\bar{T})$ is included because $f \equiv 0$ for $i>N$, if we have over-est mated the number of disks in the subgroup as $\bar{i} \rightarrow N$ when we use Eq. (57). For Gaussian weights $f_{j}$ we have

$$
\begin{gathered}
\langle Y\rangle_{m} \approx \frac{12}{N}\left(\frac{\bar{j}}{N}\right)^{2}\left(1-\frac{\bar{j}}{N}\right) H\left(1-\frac{\bar{j}}{N}\right) \cos \left(\frac{\sqrt{\frac{1}{N}}}{\sqrt{N}}\right)\left(\frac{T}{m}\right)\left(\frac{N}{N+i}\right) \\
\approx \frac{12}{m}\left(\frac{2}{4 \pi m N}\right)^{6}\left[1-\left(\frac{2}{4 \pi m N}\right)^{2}\right]\left[1+\left(\frac{2}{4 \pi m N}\right)^{2}\right]^{-2} \times \\
\times H\left(1-\left(\frac{2}{4 \pi m N}\right)^{2}\right) \cos \left(\frac{z^{2}}{4 \pi m N}\right) .
\end{gathered}
$$

To simp] iffy matters let

$$
x=\left(\frac{2}{4 \pi m N}\right)^{2},
$$

Then

$$
\langle\rangle_{m}=\frac{12}{m} x^{3} \frac{(1-x)}{(1+x)} H(1-x) \cos (4 \pi m N x) .
$$

The envelope maximum occurs at

$$
x_{0}=.721
$$

which corresponds to

$$
z_{0}=4 \pi \mathrm{Nm} \sqrt{x_{0}}=10.67 \mathrm{~m} \mathrm{~N} .
$$


For $N=30$ the predicted regurgitation maxima occur at

$$
z_{0}(m=1,2,3)=320,640,960 \text {, }
$$

in excellent agreement with the computed envelope. The first maximuin for $N=100$ also appears in agreement with Eq. (64).

A second prediction is the amplitude of $\langle\varphi\rangle$ at the maximum. Eq. (62) yields

$$
\left\langle Y\left(x_{0}\right)\right\rangle_{m} \approx \frac{.729}{m}
$$

which is large by a factor of 7.75 for the first maximum of the $N=30$ case, but is in fair agreement with its second and third maximum. The computed first maximum for $N=100$ is low by at least a factor of 3 compared with Eq. (66). Hence the amplitude of regurgitation cannot be regarded as understood at this time. The low amplitude of the first damped minimum is not understood eitier, but it may be viewed as a benefit that is paid for by regurgitation. Essentially, $/ \sqrt{\mathbb{N}}$ noise behayior is delayed to very large $z$ for both phenomena. A third abservation is the increasing frequency within the regurgitation envelope as a maximum is approached. This is clearly predicted -- see Eq. (60), and results from the dependence of $\bar{i}$ \& $z^{2}$.

\section{Acknowledgement}

This report collects and develops the ideas of a number of people, at least some of whom are the collaborators mentioned in the text. Numerical results used here were obtained by $A$. Cole. An independent analysis of the Gaussian weight function and its implementation in EMPULSE were accomplished by W. Fawley. 


\section{Appendix}

An Alternate Gaussian Weight Function

As mentioned in Section VI, the function

$$
f=2 \pi \quad(0<\pi<1)
$$

is a satisfactory candidate for the Gaussian weight if we do not impose the condition $f(n+1)+0$. In the interests of completeness the resultant dispersion relation is given here. We have

$$
\begin{aligned}
& -i \omega \tau_{1}=\int_{0}^{1} d n \frac{2 n s}{\pi-s} \\
& =2 s\left\{1+s\left[\pi i+\sin \left(\frac{1-s}{s}\right)\right]\right\} .
\end{aligned}
$$

The maximum growth rate occurs at

$$
s_{0}=.5000,
$$

with

$$
\begin{aligned}
& I_{m}\left(\omega_{0} \tau_{1}\right)=1.0000, \\
& \left(\frac{d Z}{d \tau}\right)_{0}=\frac{8.89}{\tau_{l} k_{m}} .
\end{aligned}
$$

Comparison with the Bennett gives

$$
\begin{aligned}
& \frac{\left(I_{m{ }_{0}}\right)^{G}}{\left(I_{m} \omega_{0}\right)^{B}}=1.26 \\
& \frac{(d z / d \tau)^{G}}{(d z / d \tau)^{B}}=1.22
\end{aligned}
$$

This case thus lies between the Bennett and Gaussian weight previously analyzed in this report. 
1. E. P. Lee, Kinetic Theory of a Relativistic Beam, P. F. 19, No. 1, 1976.

2. E. J. Lauer, E. P. Lee, R. J. Briggs, R. E. Hester, and R. L. Spoerlein, Radial Expansion of a Self-Focused Relativistic Electron Beam, P.F. Ig, No. 7, 1976.

3. E. P. Lee, Resistive Hose Instability of a Beam with the Bennett Profile, P. F. 21, No. 8, 1978,

4. F. W. Chambers, private communication.

5. E. P. Lee, F. W. Chambers, L. L. Lodestro, S. S. Yu, UCRL 79886, Stable Propagation of an Electron Bearn in Gas, Proc, of the 2nd Int'l Topical Conference on High Power Electron and Ion Beam Research and Technology held at Cornel? iniv., Ithaca, Ni, Oct. 3 - 5, 1977. 


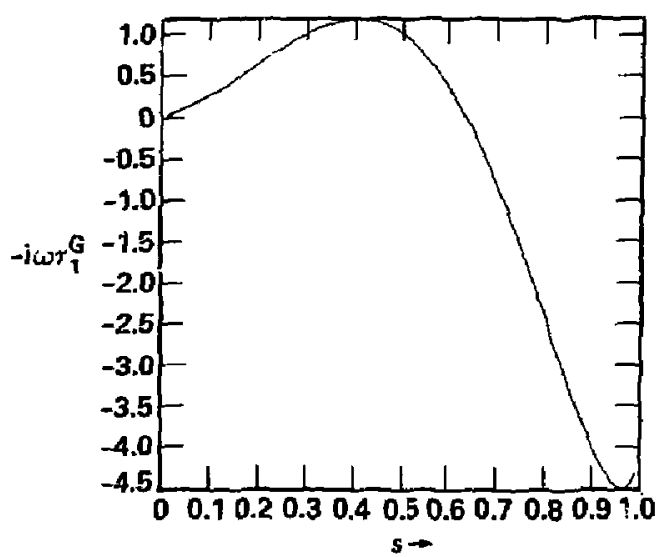

Fig. 1. Growth rate for given real $\Omega$ 
$-24-$

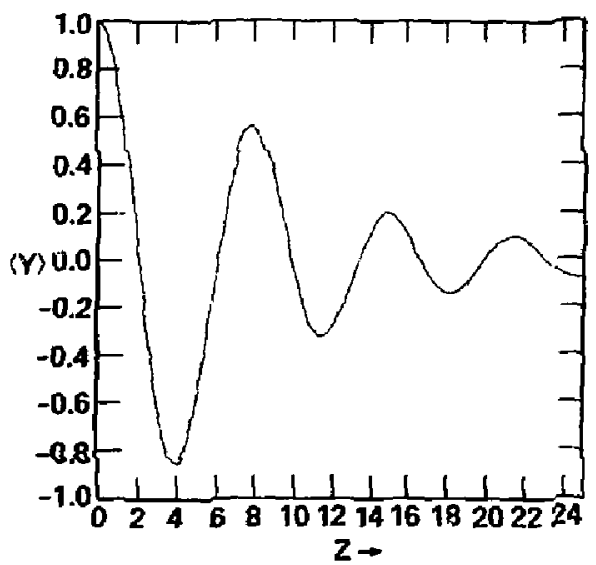

Fig. 2. Phase inix damping of a single perturbed segment - the continuous limit of the spread mass moctel. 


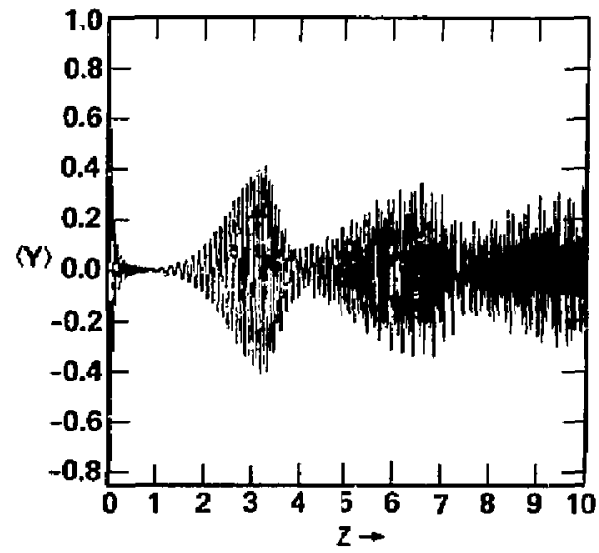

Fig. 3. Damping for $\mathbf{N}=30$ 


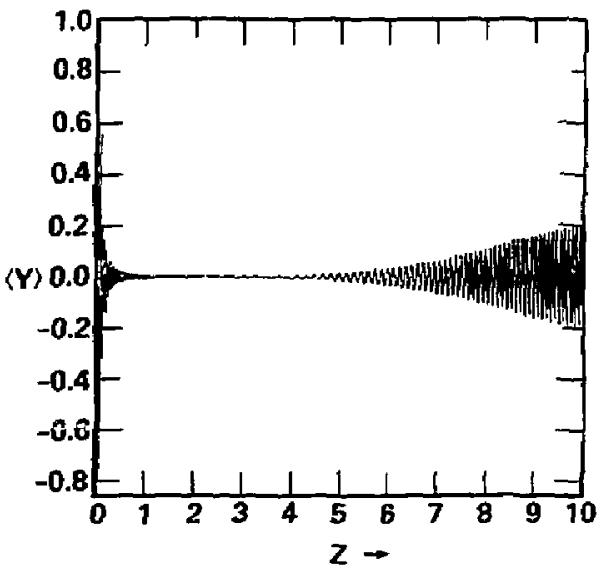

Fig. 4. Damping for $\mathbf{N}=100$ 